\title{
Transcriptional Regulation of Voltage-Gated Sodium Channels Contributes to GM-CSF-Induced Pain
}

\author{
Fan Zhang, ${ }^{1,2,3,4 \star}$ Yiying Wang, ${ }^{1,3,4 \star}$ Yu Liu, ${ }^{1,2,3,4}$ Hao Han, ${ }^{1,3,4}$ Dandan Zhang, ${ }^{2}$ Xizhenzi Fan, ${ }^{2}$ Xiaona Du, ${ }^{1,3,4}$ \\ Nikita Gamper, ${ }^{1,3,4,5}$ and Hailin Zhang ${ }^{1,3,4}$ \\ Departments of ${ }^{1}$ Pharmacology, ${ }^{2}$ Biochemistry and Molecular Biology, ${ }^{3}$ Key Laboratory of Neural and Vascular Biology, Ministry of Education, Hebei \\ Medical University, Shijiazhuang 050017, People's Republic of China, ${ }^{4}$ Key Laboratory of New Drug Pharmacology and Toxicology, Hebei Province, \\ Shijiazhuang 050017, People's Republic of China, and ${ }^{5}$ School of Biomedical Sciences, Faculty of Biological Sciences, University of Leeds, Leeds LS2 9JT, \\ United Kingdom
}

Granulocyte-macrophage colony-stimulating factor (GM-CSF) induces the production of granulocyte and macrophage populations from the hematopoietic progenitor cells; it is one of the most common growth factors in the blood. GM-CSF is also involved in bone cancer pain development by regulating tumor-nerve interactions, remodeling of peripheral nerves, and sensitization of damage-sensing (nociceptive) nerves. However, the precise mechanism for GM-CSF-dependent pain is unclear. In this study, we found that GM-CSF is highly expressed in human malignant osteosarcoma. Female Sprague Dawley rats implanted with bone cancer cells develop mechanical and thermal hyperalgesia, but antagonizing GM-CSF in these animals significantly reduced such hypersensitivity. The voltage-gated Na ${ }^{+}$ channels Nav1.7, Nav1.8, and Nav1.9 were found to be selectively upregulated in rat DRG neurons treated with GM-CSF, which resulted in enhanced excitability. GM-CSF activated the Janus kinase 2 (Jak2)-signal transducer and activator of transcription protein 3 (Stat3) signaling pathway, which promoted the transcription of Nav1.7-1.9 in DRG neurons. Accordingly, targeted knocking down of either Nav1.7-1.9 or Jak2/Stat3 in DRG neurons in vivo alleviated the hyperalgesia in male Sprague Dawley rats. Our findings describe a novel bone cancer pain mechanism and provide a new insight into the physiological and pathological functions of GM-CSF.

Key words: bone cancer pain; DRG; GM-CSF; Jak2-Stat3; neural excitability; voltage-gated sodium channels

\section{Significance Statement}

It has been reported that granulocyte-macrophage colony-stimulating factor (GM-CSF) plays a key role in bone cancer pain, yet the underlying mechanisms involved in the GM-CSF-mediated signaling pathway in nociceptors is not fully understood. Here, we showed that GM-CSF promotes bone cancer-associated pain by enhancing the excitability of DRG neurons via the Janus kinase 2 (Jak2)-signal transducer and activator of transcription protein 3 (Stat3)-mediated upregulation of expression of nociceptorspecific voltage-gated sodium channels. Our study provides a detailed understanding of the roles that sodium channels and the Jak2/Stat3 pathway play in the GM-CSF-mediated bone cancer pain; our data also highlight the therapeutic potential of targeting GM-CSF.

\section{Introduction}

Granulocyte-macrophage colony-stimulating factor (GM-CSF) was originally identified as a colony-stimulating factor because of

\footnotetext{
Received Aug. 26, 2018; revised April 12, 2019; accepted April 15, 2019.

Author contributions: F.Z., X.D., N.G., and H.Z. designed research; F.Z., Y.W., Y.L., H.H., D.Z., and X.F. performed research; F.Z. analyzed data; H.Z. wrote the paper.

${ }^{*}$ F.Z. and Y.W. contributed equally to this work

The authors declare no competing financial interests.

This work is supported by the National Natural Science Foundation of China (Grant 91732108 to H.Z.; Grants 31872788 and 31401199 to F.Z.; and Grant 31571088 to X.D.); the Key Basic Research Project of Applied Basic Research Program of Hebei Province (Grant $16967712 D$ to X.D.); and the Biotechnology and Biological Sciences Research Council (Grants BB/R003068/1 and BB/R02104X/1 to N.G.). We thank Yueping Liu (The Fourth Hospital of Hebei Medical University) for the collection of human osteosarcoma and chondroma tissues.

Correspondence should be addressed to Hailin Zhang at zhangh@@ebmu.edu.cn.
}

its ability to induce granulocyte and macrophage populations from precursor cells. GM-CSF is also abundantly secreted by some tumor cells and plays a key role in regulating tumor-nerve interactions, remodeling of peripheral nerves, and sensitization of damage-sensing nerves (nociceptors) by acting at its receptors (Schweizerhof et al., 2009). Apart from the bone metastases pain, GM-CSF was also shown to be involved in inflammatory, arthritic, and neuropathic pain (Cook et al., 2012, 2013; Nicol et al., 2018). A recent study (Nicol et al., 2018) show that GM-CSF signaling contributes to pain-associated behavior that is indepen- 
dent of a gliosis and/or astrocyte response, suggesting that GMCSF may directly activate sensory neurons. However, the precise mechanism for GM-CSF-dependent pain is unclear.

Dorsal root ganglion (DRG) neurons are the peripheral somatic and visceral sensory neurons; a subset of these neurons is responsible for nociceptive signal initiation and propagation. GM-CSF receptors (GM-CSFRs) are found to be expressed in DRGs and in peripheral nerves dispersed in the periosteum of mice (Schweizerhof et al., 2009). Signaling cascades and mechanisms of action of GM-CSFRs in sensory neurons are largely unknown, but in hematopoietic cells, activation of GM-CSFRs is known to stimulate cell-signaling pathways regulating gene expression, including the Janus kinase (Jak)-signal transducer and activator of transcription protein (Stat) pathway (Stosser et al., 2011). The activation of Jak leads to dimerization and translocation of Stat family transcription factors to cell nucleus to regulate gene expression (Fortin et al., 2007). The main aim of the present study was to identify molecules involved in the GM-CSFmediated signaling pathway in nociceptors and test their relevance to GM-CSF-induced pain.

Ion channels are the basis of sensory neuronal excitability and were previously suggested as molecular targets of GM-CSF signaling pathway (Bali et al., 2013). Our preliminary screening (see Results; Figs. 1, 2, Fig. 3) revealed that GM-CSF selectively increased expression in DRG neurons of three voltage-gated sodium channels: Nav1.7, Nav1.8, and Nav1.9. Due to the primary role of these channels in the ability of DRG neurons to generate action potentials (APs), we hypothesized that GM-CSF might promote pain and hyperalgesia by acting on voltage-gated $\mathrm{Na}^{+}$ channels in nociceptors.

At least five different voltage-gated sodium channels are reportedly expressed in DRG, including the TTX-sensitive Nav1.1, Nav1.6, and Nav1.7, and the TTX-resistant Nav1.8 and Nav1.9 (Cummins et al., 2000). Nav1.7, Nav1.8, and Nav1.9 channels are mainly distributed in small-diameter DRG neurons, most of which are involved in nociception. Nav1.7 produces a rapidly activating and inactivating but slowly repriming current. It produces a robust ramp current in response to depolarizations, contributing to the generation and propagation of action potentials and acting as a threshold channel regulating excitability (François-Moutal et al., 2018; Li et al., 2018). Gain-of-function mutations within the Nav1.7 gene SCN9A lead to inherited pain disorders, such as erythromelalgia and paroxysmal extreme pain disorder (Dib-Hajj et al., 2008; Jarecki et al., 2010; Cheng et al., 2011). Nav1.8 mediates a slowly inactivating sodium current acting as a key component of the upstroke of the action potential, and thus influences neuronal excitability and nociceptive transmission. Mutations of the Nav1.8 gene SCN10A is found in patients with peripheral neuropathy (Lai et al., 2002; Choi et al., 2007; Blanchard et al., 2012). The Nav1.9 channel has slow kinetics and is responsible for persistent $\mathrm{Na}^{+}$currents in nociceptors; together with the Nav1.7, it acts as a threshold channel for AP firing; it amplifies subthreshold stimuli leading to AP bursts (Huang et al., 2014). Gain-of-function mutations of the Nav1.9 channel gene SCN11A cause familial episodic pain syndrome (Huang et al., 2014, 2017).

In this study, we found that GM-CSF significantly increased the excitability of DRG neurons in parallel with the increase in the current density; mRNA; and protein expression of Nav1.7, Nav1.8, and Nav1.9 channels. We further show Jak2-Stat3mediated upregulation of Nav1.7, Nav1.8, and Nav1.9 channel expression in nociceptors is a major factor in the GM-CSFrelated component of bone cancer pain.

\section{Materials and Methods}

Human subjects. The study was performed in accordance with the ethical principles for medical research involving human subjects set out in the Helsinki Declaration, and was approved by the ethical committee at Hebei Medical University (Shijiazhuang, People's Republic of China). Osteosarcoma or chondroma tissues were obtained from eight patients from the Fourth Hospital of Hebei Medical University. Each specimen was fixed with $4 \%$ paraformaldehyde for immunohistochemistry study. All patients or their relatives gave informed consent before their participation in the study.

Animals. The animal protocols used in this study were approved by the Animal Care and Ethical Committee of Hebei Medical University under the International Association for the Study of Pain guidelines for animal use. All surgeries were performed under sodium pentobarbital (SigmaAldrich) anesthesia, and all efforts were made to minimize animal suffering.

Rat DRG neuron culture. DRG neurons were obtained from adult Sprague Dawley rats (provided by the Experimental Animal Center of Hebei Province, People's Republic of China) based on the protocol described previously (Du et al., 2014). Briefly, the ganglia were digested at $37^{\circ} \mathrm{C}$ with collagenase $(2 \mathrm{mg} / \mathrm{ml})$ with dispase $(7.5 \mathrm{mg} / \mathrm{ml})$ for $30 \mathrm{~min}$. Ganglia were then mechanically triturated and washed twice with DMEM supplemented with $10 \%$ fetal calf serum. Thereafter, the DRG neurons were plated on poly-D-lysine-coated glass coverslips.

Quantitative PCR. Total RNA was extracted using a commercial RNA isolation kit (RNAiso, Takara). Isolated RNA was dissolved in $20 \mu \mathrm{l}$ of DEPC-treated water and reverse-transcribed using a reverse transcription reagent kit (PrimeScript RT Reagent Kit with gDNA Eraser, Takara) and a thermal cycler (Mastercycler, Eppendorf). Quantitative PCR (qPCR) was performed using a kit [SYBR Premix Ex TaqII (Tli RNase H Plus), Takara], and the fluorescent DNA was detected and quantified with an FQD-48A(A4) system (BIOER). The PCR products were also run on a $2 \%$ agarose gel and were visualized using a gel imager (TFP-M/WL, Vilber Lourmat). For qPCR analysis, the following specific primers were used: Nav1.7: forward-GCTCCAAGGACACAAAACGAAC; reverseATCAGACTCCCCAGGTGCAAT; Nav1.8: forward-GACCCTTTCTA CAGCACACACC; reverse-AAGTCCAGCCAGTTCCACG; Nav1.9: forward-GCCCCTTCACTTCCGACT; reverse-GTCTTCCAGAGGCT TCGCTAC; and GAPDH: forward-CCAGCCTCGTCTCATAGACA; reverse-CGCTCCTGGAAGATGGTGAT.

Luciferase reporter assay. The Stat3 luciferase reporter vector was designed to measure the binding of transcription factors to the enhancer and was transfected into HEK293 cells with Lipofectamine 2000 reagent (Invitrogen).

Fragments of rat $\operatorname{Scn} 9 a, \operatorname{Scn} 10 a$, and $\operatorname{Scn} 11 a$ genes were amplified by PCR with the following primers: corresponding to the promotor region ofS $c n 9 a(-286 /+306)$ :forward-GGCTCGAGAGCTTAAGGAAAGGAG GGTA, reverse-GTAAGCTTTTTCCCCTTTGACTCCTTAC; corresponding to the promotor region of Scn10a $(+749 /+124)$ : forwardGGCTCGAGCCGTAGTAAGACCCTGCCTTG, reverse-GTAAGCTT GAGACCCCAGCTCTGCAAAAC; and corresponding to the promotor region of $\operatorname{Scn} 11 a$ ( $-51 /+556$ regions): forward-GGCTCGAGCTTCACA TGGTTGATCCATC, reverse-GTAAGCTTATTCTCGCTCTTGGCAGTA.

Amplified fragments were digested with appropriate restriction enzymes and cloned into pGL3 Basic (Promega). Luciferase activity was measured using a Dual Luciferase Assay Kit (Promega). Specific promoter activity was expressed as the relative activity ratio of firefly luciferase to Renilla luciferase.

Western blot. The DRG neuron lysates were prepared with RIPA lysis buffer. Equal amounts of protein were separated by SDS-PAGE and electrotransferred to a polyvinylidene fluoride membrane. Membranes were blocked with $5 \%$ nonfat dairy milk and incubated with primary antibodies against Nav1.7 (1:500; Alomone Labs), Nav1.8 (1:500; Abcam), Nav1.9 (1:200; Abcam), phospho (p)-Jak1 (1:1000; Affinity), p-Jak2 (1: 1000; Affinity), p-Jak3 (1:1000; Affinity), p-stat3 (1:1000; Epitomics), and p-Stat5a (1:1000; Affinity) at $4{ }^{\circ} \mathrm{C}$ overnight. This was followed by incubation with IRDye800-conjugated secondary antibody $(1: 20,000$; 


\section{A}
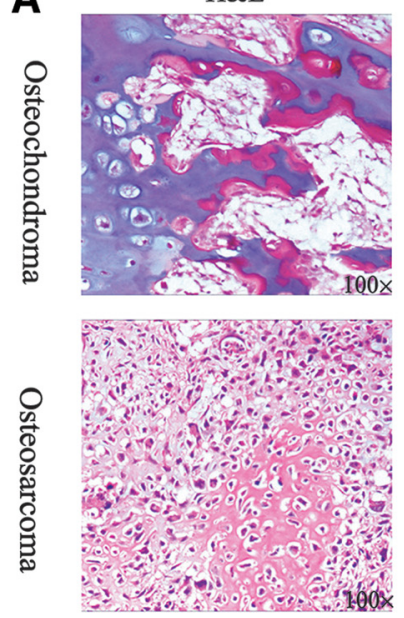

Anti-GM-CSF
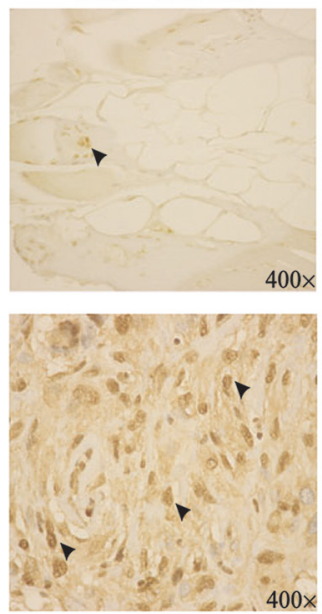

B

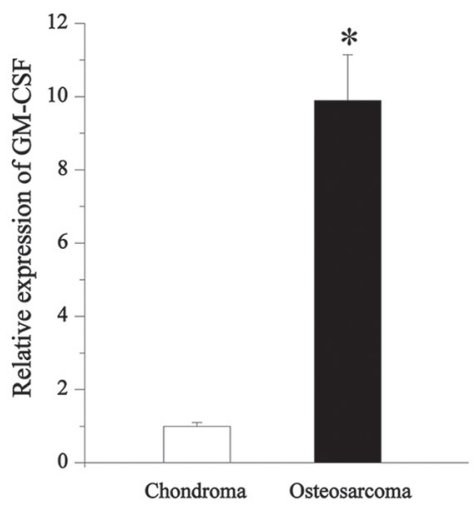

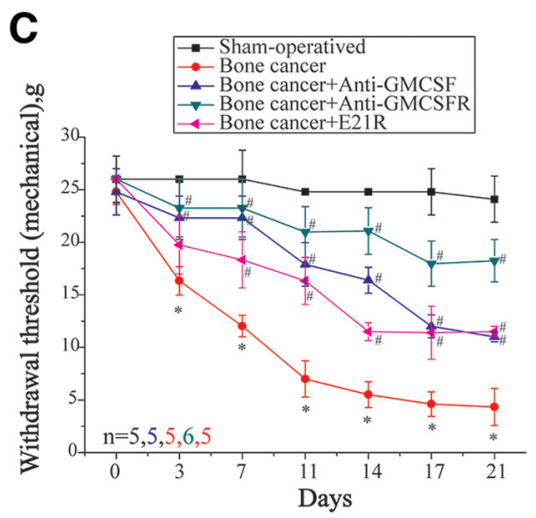

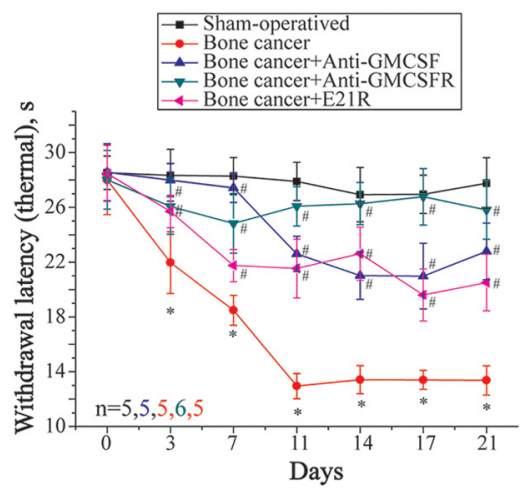

D
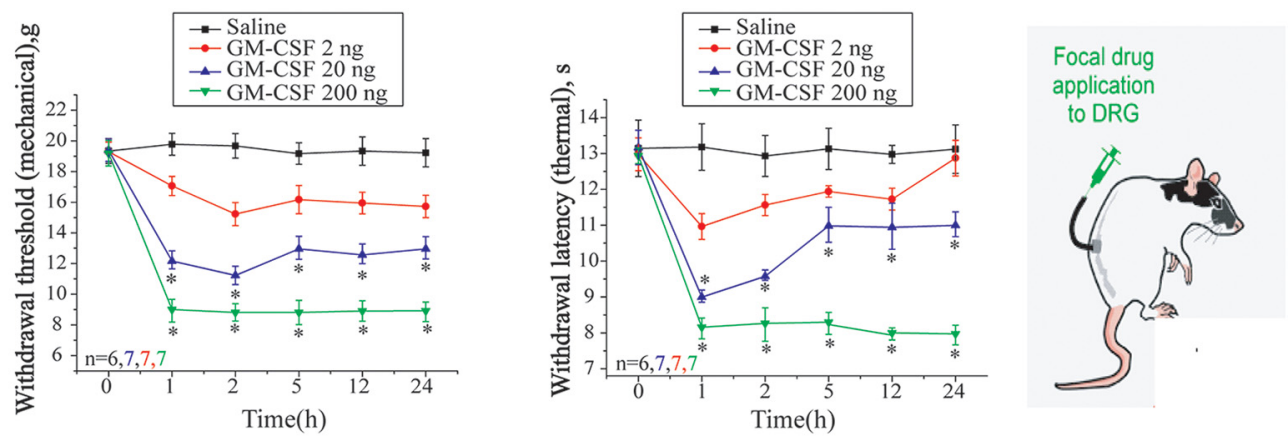

Figure 1. Role of GM-CSF in bone cancer metastases pain. $A$, High expression level of GM-CSF in osteosarcoma tissue sample. H\&E staining of chondroma and osteosarcoma is shown on the left; immunohistochemical staining for GM-CSF, indicated by arrows in chondroma and osteosarcoma, is shown on the right. $B$, Summary results for immunohistochemical staining for GM-CSF $(n=$ 8/group; unpaired $t$ test: $\left.t=5.69,{ }^{*} p=0.0013\right)$. C, Effect of antibodies against GM-CSF or GM-CSFR (10 $\mu \mathrm{g}$ ) and GM-CSF analog E21R (a competitive antagonist of GM-CSF, $25 \mu \mathrm{g} / \mu \mathrm{l}, 3 \mu \mathrm{l}$ ) on mechanical (left) and thermal (right) nociceptive responses in a bone cancer pain model in rats. The mechanical paw withdrawal threshold and thermal paw withdrawal latency were measured at $3,7,11,14,17$, and $21 \mathrm{~d}$ for the control group (black line + squares), the bone cancer group (red line + circles), the bone cancer + antibody against GM-CSF group (blue line + triangles), the bone cancer + antibody against GM-CSFR group (pink line + triangles), and the bone cancer + E21R group (green line + triangles). Two-way ANOVA followed by Bonferroni post hoc tests revealed a significant effect of treatment $\left(F_{(4,150)}=29.49, p=0\right)$ and time $\left(F_{(5,150)}=5.06, p<0.0001\right)$, but no interaction between the two $\left(F_{(20,150)}=0.71, p=0.81\right.$; left); and a significant effect of treatment $\left(F_{(4,240)}=15.10, p<0.0001\right)$, time $\left(F_{(4,240)}=2.56, p=0.028\right)$, and an interaction between the two $\left(F_{(20,240)}=2.20, p=0.003\right.$; right). ${ }^{*} p<0.05$ compared with sham group; $\# p<$ 0.05 with respect to the corresponding bone cancer group. (D) Dose-dependent effects of GM-CSF on paw withdrawal threshold to mechanical stimulus (left) and on paw withdrawal latency to noxious heat (right) at 1,2,5,12, and $24 \mathrm{~h}$ following focal DRG application via DRG cannula. Number of experiments is indicated as $n$ in each panel. Two-way ANOVA followed by Bonferroni post hoc tests revealed a significant effect of dose $\left(F_{(3,345)}=29.30, p<0.0001\right)$ but not of time $\left(F_{(3,345)}=0.15, p=0.96\right)$ or interaction between the two $\left(F_{(12,345)}=0.37, p=0.97 ;\right.$ left). Right, A significant effect of dose $\left(F_{(3,350)}=70.95, p=0\right)$ and an interaction between dose and time $\left(F_{(12,350)}=3.71, p<0.0001\right)$, but the effect of time did not reach significance $\left(F_{(3,350)}=2.09, p=\right.$ $0.08) .{ }^{*} p<0.05$ compared with the vehicle saline.

Rockland) for $1 \mathrm{~h}$ at room temperature and subsequent scanning with the Odyssey Infrared Imaging System (LI-COR). The integrated intensity for each detected band was determined using Odyssey Imager software (version 3.0).
Immunohistochemistry. Sections from human osteosarcoma or chondroma tissues were blocked with $0.3 \%$ hydrogen peroxide, followed by preincubation with $5 \%$ normal goat serum and then incubation with primary antibodies against GM-CSF (1:200; PeproTech) at $4^{\circ} \mathrm{C}$ over- 
A

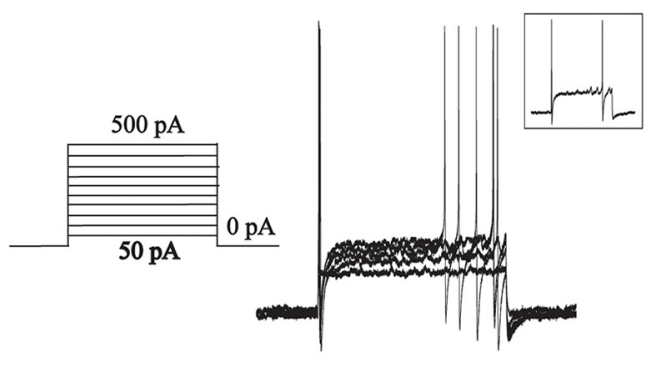

Control

C

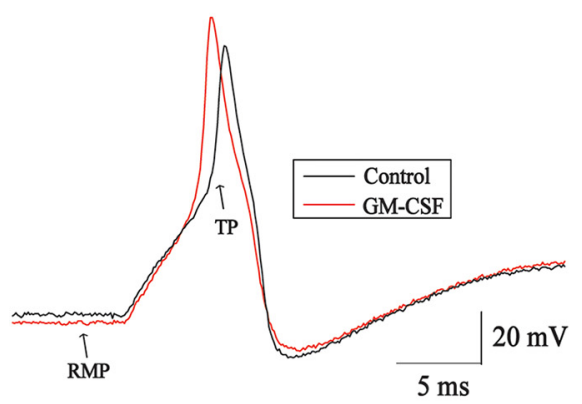

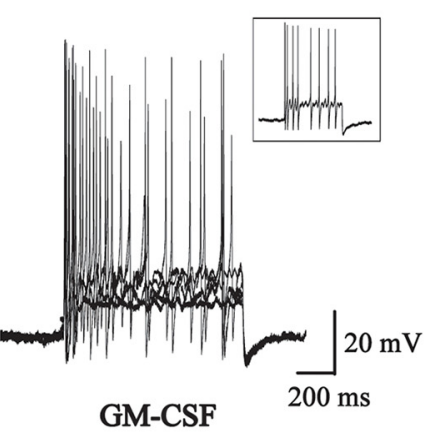

GM-CSF

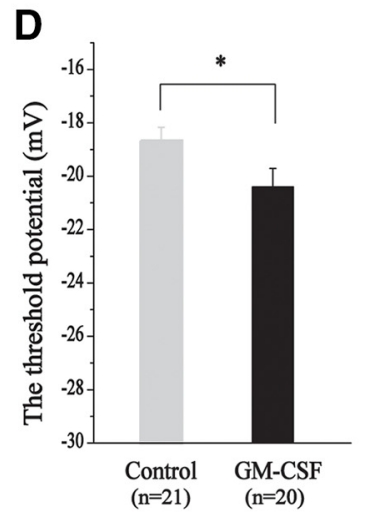

B

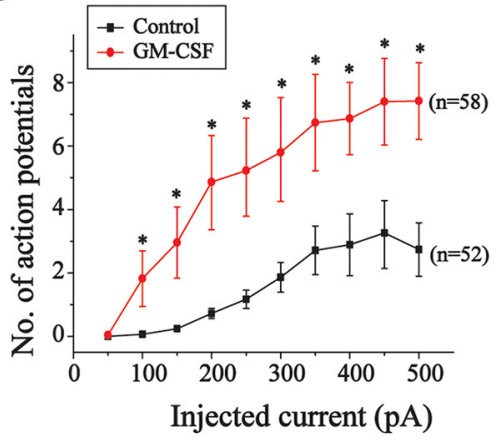

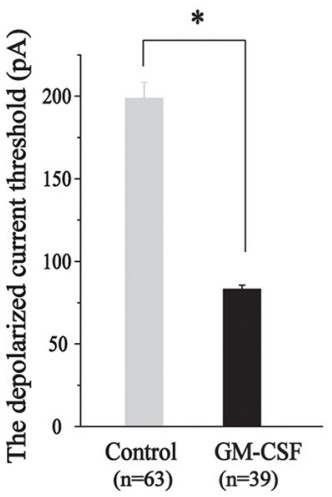

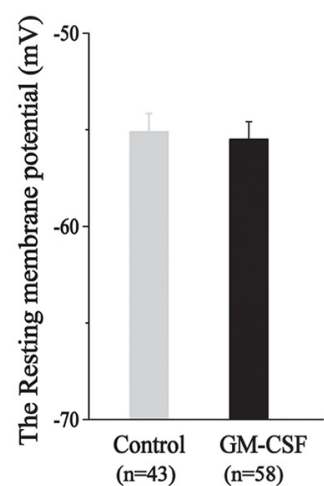

Figure 2. Effect of GM-CSF on the excitability of small-sized DRG neurons. $A$, Representatives of action potentials evoked by depolarizing current pulse (left), recorded from small-sized DRG neurons. $\boldsymbol{B}$, Summary results for the effect of GM-CSF on numbers of action potential induced by increasing amplitudes of depolarizing currents. Two-way ANOVA followed by Bonferroni post hoc tests revealed a significant effect of treatment $\left(F_{(1,1057)}=22.45, p<0.0001\right)$ and injected currents $\left(F_{(9,1057)}=7.82, p<0.0001\right)$, but not of a significant interaction between the two $\left(F_{(9,1057)}=\right.$ $0.43, p=0.92) .{ }^{*} p<0.05$ compared with the control. $C$, Single action potentials from $A$ with expanded time scales. $D$, Summary results for the effect of GM-CSF on the threshold potential, rheobase current, and RMP (unpaired $t$ test, ${ }^{*} p<0.05$ compared with the control).

night. Next, the sections were incubated with the biotinylated secondary antibody, followed by streptavidin-horseradish peroxidase and diaminobenzidine, and then counterstained with hematoxylin. Staining intensities were determined by measurement of the integrated optical density by light microscopy using a computer-based Image-Pro Morphometric System.

Electrophysiology. Action potentials were recorded from dissociated rat small-diameter DRG neurons $(<25 \mu \mathrm{m})$ under current clamp using an Axopatch 200B amplifier and a Digidata 1322A converter (Molecular Devices). Pipettes (3-4 M $\Omega$ ) were filled with solution containing the following (in mM): $\mathrm{KCl} \mathrm{150,} \mathrm{MgCl}_{2}$ 5, and HEPES 10, at $\mathrm{pH} 7.4$ adjusted with $\mathrm{KOH}$. The bath solution contained the following (in $\mathrm{mM}$ ): $\mathrm{NaCl} \mathrm{160,}$ $\mathrm{KCl} 2.5, \mathrm{MgCl}_{2} 1, \mathrm{CaCl}_{2}$ 2, glucose 10, and HEPES 20, at $\mathrm{pH} 7.4$ adjusted with $\mathrm{NaOH}$. Small DRG neurons were examined for evoked activity with a series of $1 \mathrm{~s}$ current injections from 0 to $500 \mathrm{pA}$ in $50 \mathrm{pA}$ increments. The rheobase currents were determined by the first action potential elicited by a series of depolarizing current injections that increased in $5 \mathrm{pA}$ increments. The following values were measured in this study, as follows: resting membrane potential (RMP), threshold potential (TP), AP amplitude, and depolarization rate $(\mathrm{V} / \mathrm{s})$.

Sodium currents were recorded from these small-diameter DRG neurons under the voltage-clamp mode in a whole-cell configuration. Pipettes (3-4 M $\Omega$ ) were filled with solution containing the following (in mm): $70 \mathrm{CsCl}, 30 \mathrm{NaCl}, 30$ TEA-Cl, 10 EGTA, $1 \mathrm{CaCl}_{2}, 2 \mathrm{MgCl}_{2}, 2$ $\mathrm{Na}_{2}$ ATP, 0.05 GTP, 10 HEPES, and 5 glucose, at $\mathrm{pH} 7.3$ with CsOH. The bath solution for DRG neurons was as follows (in mM): $80 \mathrm{NaCl}, 50$ choline-Cl, 30 TEA-Cl, $2 \mathrm{CaCl}_{2}, 0.2 \mathrm{CdCl}_{2}, 10$ HEPES, and 5 glucose, at $\mathrm{pH} 7.3$ with $\mathrm{NaOH}$. The acquisition rate was $20 \mathrm{kHz}$, and signals were filtered at $5 \mathrm{kHz}$. Series resistances were compensated by $80 \%$. Currents were elicited by $40 \mathrm{~ms}$ pulses from a holding potential of $-120 \mathrm{mV}$ to test potentials between -80 and $+40 \mathrm{mV}$ in $5 \mathrm{mV}$ increments. The TTXresistant (TTX-R) sodium currents (including both Nav1.9 and Nav1.8 currents) were recorded in the presence of $300 \mathrm{~nm}$ TTX in the external solution. The TTX-sensitive (TTX-S) sodium currents were obtained by digital subtraction of the TTX-R sodium currents from the total currents. Nav1.8 currents were then elicited by a prepulse of $-70 \mathrm{mV}$ for $500 \mathrm{~ms}$ before the test potentials from -80 to $+40 \mathrm{mV}$ in $5 \mathrm{mV}$ increments in the same neuron. The Nav1.9 currents were obtained by digital subtraction of the Nav1.8 currents from the TTX-R sodium currents (based on protocols from the study by Qiu et al., 2016).

Rat model of tumor-evoked pain. The Walker 256 carcinosarcoma breast cancer cells were provided by the Shanghai Cell Bank of the Chinese Academy of Sciences. Wistar rats were injected intraperitoneally with $0.5 \mathrm{ml}$ of the Walker 256 cancer cells $\left(2 \times 10^{7}\right.$ cells $\left./ \mathrm{ml}\right)$, and $6-7 \mathrm{~d}$ later ascitic fluid was extracted. Sprague Dawley rats (180-200 g) were anesthetized by intraperitoneal injection of sodium pentobarbital $(60-80 \mathrm{mg} / \mathrm{kg})$. The right leg was shaved, and the skin was disinfected with $70 \%(\mathrm{v} / \mathrm{v})$ ethanol. A 1-cm-long rostrocaudal incision was made in the skin over the lower one-third of the tibia for exposure with minimal damage to muscles and nerves. The medullary canal was approached by inserting a 23-gauge needle proximally through a hole drilled in the tibia. The needle was then replaced with a $10 \mu \mathrm{l}$ microinjection syringe containing the cells to be injected. A $5 \mu$ l volume of Walker 256 cells ( $4 \times$ $10 \% \mathrm{ml}$ ) or boiled cells (sham group) were injected into the bone cavity. After a 2 min delay to allow cells to fill the bone cavity, the syringe was removed and the hole was sealed using bone wax. In some experiments, antibody against GM-CSF (10 $\mu$ g; Santa Cruz Biotechnology), antibody GM-CSFR $(10 \mu \mathrm{g}$; Santa Cruz Biotechnology), GM-CSF antagonist, E21R ( $25 \mu \mathrm{g} / \mu \mathrm{l}, 3 \mu \mathrm{l}$; LifeTein), or vehicle was injected in the vicinity of the tibia bone. The wound was closed using 1-0 silk threads and dusted with penicillin powder. The rats were allowed unrestricted movement in their cages after recovery and their general condition was monitored during the experiment. 
A The relative expression of $\mathrm{mRNA}$
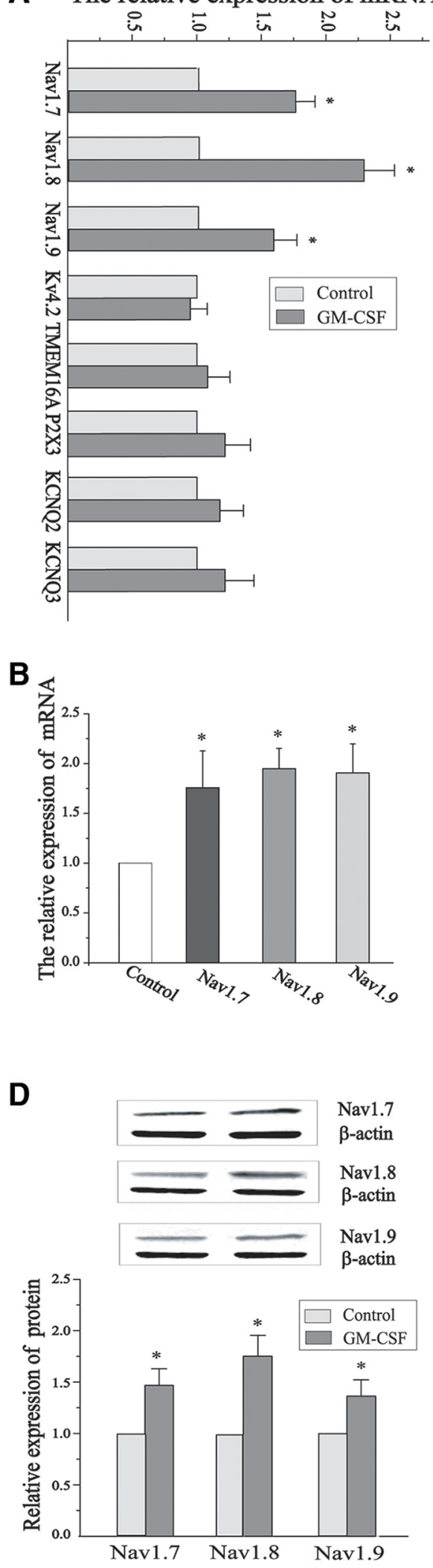

C

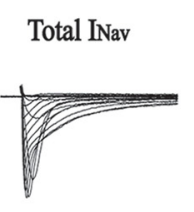

Control

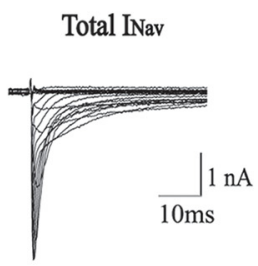

GM-CSF

TTX-S INav

TTX-S INav

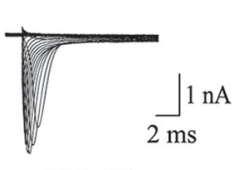

Control

GM-CSF

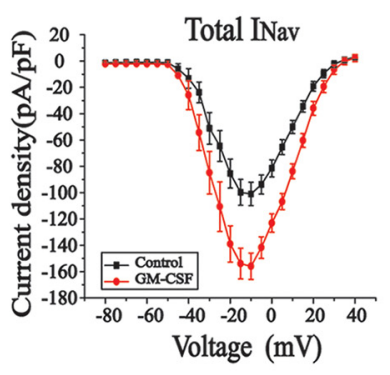

TTX-S INav
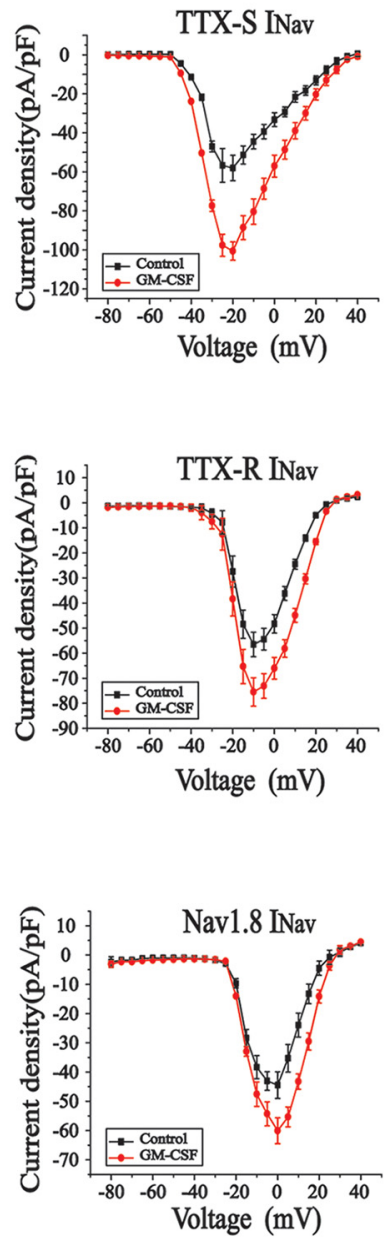

Control

GM-CSF
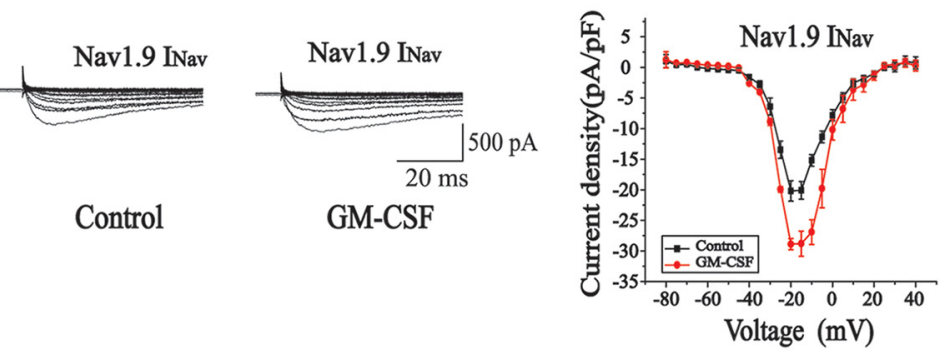

Figure 3. Effect of GM-CSF on the current amplitude and expression level of Nav1.7, Nav1.8, and Nav1.9 channels. A, Relative mRNA expression of Nav1.7, Nav1.8, Nav1.9, Kv4.2, TMEM16A, P2X3, KCNQ2, and KCNQ3 in cultured DRG cells after incubation of GM-CSF $(200 \mathrm{ng} / \mathrm{ml})$ at $24 \mathrm{~h}\left(n=9\right.$, unpaired $t$ test, ${ }^{*} p<0.05$ compared with the control). B, Relative mRNA expression of Nav1.7, Nav1.8, and Nav1.9 in DRG neurons of bone cancer pain at the seventh day. ( $n=6$, unpaired $t$ test, ${ }^{*} p<0.05$ compared with the control). C, Typical current traces and current density-voltage relationship of total TTX-S, TTX-R, Nav1.8, and Nav1.9 Na ${ }^{+}$currents in cultured DRG cells after incubation with GM-CSF (200 ng/ml) for $24 \mathrm{~h}$. D. Western blot analysis of expression levels of Nav1.7, Nav1.8, and Nav1.9 proteins in DRG neurons treated with GM-CSF $(200 \mathrm{ng} / \mathrm{ml})$ for $18 \mathrm{~h}\left(n=3\right.$, unpaired $t$ test, ${ }^{*} p<0.05$ compared with the control). 
Table 1. Animal tests and treatment types

\begin{tabular}{lll}
\hline Group & Type of ODN pretreatment & Treatment type \\
\hline 1 & Mismatched ODN & GM-CSF or saline \\
2 & AS ODN Scn9a & GM-CSF or saline \\
3 & AS ODN Scn10a & GM-CSF or saline \\
4 & AS ODN Scn11a & GM-CSF or saline \\
5 & AS ODN Jak2 & GM-CSF or saline \\
6 & AS ODN Stat3 & GM-CSF or saline \\
7 & AS ODN Stat5a & GM-CSF or saline \\
\hline
\end{tabular}

Focal application of drugs to DRG in vivo. All surgical procedures were performed under deep anesthesia with an intraperitoneal injection of pentobarbital sodium $(60-80 \mathrm{mg} / \mathrm{kg})$. A DRG cannula for focal application of substances to DRG was implanted as previously described (Du et al., 2017). Briefly, a midline incision was made at the L4-L6 spinal level of adult male rats (Sprague Dawley; weight, $180-200 \mathrm{~g}$ ), and the L5 was identified at the midpoint of a link between both sides of the iliac crest. A $0.8 \mathrm{~mm}$ hole ( $\sim 1 \mathrm{~mm}$ off the inferior edge of the transverse process) was drilled through the transverse process over the L5 DRG. Approach of a ganglion was verified by the twitch of the paw. A hooked stainless steel blunt-tip cannula (inner diameter, $0.64 \mathrm{~mm}$; length, $4 \mathrm{~mm}$ ) was forced into the hole and connected to a polypropylene tube (inner diameter, $0.41 \mathrm{~mm}$; length, $4.5 \mathrm{~mm}$ ). The incision was closed with sutures, and the cannula was firmly fixed in place with dental cement. Intramuscular injection of benzylpenicillin $(19 \mathrm{mg} / 0.1 \mathrm{ml})$ was given immediately after surgery. Postoperatively, rats were housed individually in plastic cages with sawdust flooring and supplied with water and food ad libitum. Animals were left to recover for at least $24 \mathrm{~h}$ before the experiments were performed. Animals developing signs of distress were humanely killed.

Antisense oligonucleotide knockdown. On the second day after DRG cannula implantation, rats were given through the cannula the antisense (AS) oligodeoxynucleotides (ODNs) against $S c n 9 a, S c n 10 a, S c n 11 a$, Jak2, Stat3, or Stat5 (each at $12.5 \mu \mathrm{g}$ in $5 \mu \mathrm{l}$ ). The AS ODNs were given consecutively twice a day for $4 \mathrm{~d}$. Mismatched ODNs were also given at matched time points. On the fifth day, mechanical and thermal sensitivities were assessed at 1, 2, 5, 12, and $24 \mathrm{~h}$ after the focal DRG application of GM-CSF $(5 \mu \mathrm{l})$ or saline $(5 \mu \mathrm{l})$, respectively. Seven groups of animals were tested and treated (Table 1).

The following specific AS ODNs were used: mismatched ODN: TCACCCAGCACCCCCAACACATAGTT; Scn9a: CTGGATCAACA TGGTCTTCA; Scn10a: CCAGAACCAAGCACAGAGGA; Scn11a: CA CCATCTGCATCATCATCA; Jak2: AAGGTACAGATTCCGCAGGT; Stat3: ACATGGAGGAGTCCAACAAC; and Stat5a: TCTCAGTTCA GCGTTGGCAG.

Mechanical hyperalgesia. Threshold sensitivity to mechanical stimuli was assessed using the von Frey method, as described previously (Chaplan et al., 1994). Briefly, calibrated nylon filaments (von Frey hair, Stoelting) with different bending forces were applied to the midplantar surface of the right hindpaw of the rats. The filaments were applied starting with the softest and continuing in ascending order of stiffness. A brisk withdrawal of the right hindlimb was considered a positive response.

Thermal hyperalgesia. The paw withdrawal latency in response to heat was tested using the Hargreaves method on the right hindpaw of the rats with a radiant heat lamp source (Mengtai Technology). The intensity of the radiant heat stimulus was maintained at $20 \%$. The time to withdrawal of the right hindpaw (elapsed time) was recorded.

Blinding and randomization. In all in vivo experiments when animal behavior tests and drug administration were involved, experiments were conducted on the basis of a blind and randomized design. To achieve this, two experimenters performed every test: one experimenter was in charge of drug injections and group randomization; and the second experimenter, blinded to the drug administration and grouping schedules, conducted the mechanical and thermal sensitivity measurements.

Statistics and analysis. All data are given as the mean \pm SEM. Differences between groups were assessed by paired or unpaired $t$ test. Comparisons of the behavioral data between groups at each individual time point were conducted using a two-way ANOVA followed by Bonferroni post hoc tests. Comparisons of the number of action potentials between groups at each injected current were conducted using a two-way ANOVA followed by Bonferroni post hoc tests. Differences were considered significant at $p \leq 0.05$. Statistical analyses were performed using OriginPro 9.1 (OriginLab).

\section{Results}

\section{GM-CSF plays a crucial role in bone metastases cancer pain}

Osteosarcoma is the most common form of primary bone cancer, in which pain is the most common symptom and is seen in $85 \%$ of patients (Yoneda et al., 2015). Osteochondroma is the most common benign bone tumor, and the majority of osteochondromas form an asymptomatic, hard, immobile, painless palpable mass (de Mooij et al., 2012). We first collected tumor tissue from osteosarcama and osteochondroma patients after surgery, and compared the expression levels of GM-CSF in these two tumor tissue types. The $\mathrm{H} \& \mathrm{E}$ staining shows typical characteristics of cancerous (Fig. 1A, bottom left) and benign (Fig. 1A, top left) bone tissue. Interestingly, osteosarcoma biopsy samples demonstrated dramatically increased levels of GM-CSF compared with those seen in osteochondroma samples, as revealed with immunohistochemistry (Fig. $1 A$, right panels, $B ; n=8,{ }^{\star} p<0.05$ ).

To explore the role of GM-CSF in bone cancer pain, we first established the bone metastases cancer pain model induced by the implanted Walker 256 carcinoma cells. Consistent with previous reports (Wang et al., 2011), mice with tumors in the tibia bone displayed significantly lower withdrawal thresholds for mechanical stimuli and shortened withdrawal latencies for thermal stimuli at 3-21 d after tumor cell injection (Fig. $1 C, D$ ). To assess the role of GM-CSF in these behavioral manifestations of pain hypersensitivity, we injected a mutant GM-CSF peptide with the glutamate-to-arginine substitution at position 21 of a rat GMCSF peptide sequence (E21R). E21R acts as a competitive antagonist of GM-CSF and can neutralize some of its biologic actions (Iversen et al., 1996); however, its effect on GM-CSF-mediated pain has not been tested before. E21R was injected in the vicinity of the tibia bone where the cancer cells had been implanted (see Materials and Methods). Treatment of the bone cancer rats with E21R significantly alleviated both mechanical and thermal hyperalgesia, respectively (Fig. $1 C$ ). The reduction of hyperalgesia was registered starting from day 7 after the establishment of the bone cancer model and lasted for the duration of observation $(21 \mathrm{~d}$ ). To further validate the role of GM-CSF in the bone cancer pain, we also used antibodies against GM-CSF and GM-CSFR. The injection of anti-GM-CSF and anti-GM-CSFR antibodies also produced a strong anti-hyperalgesic effect, with the anti-GMCSFR antibody being particularly efficacious, resulting in significantly stronger anti-hyperalgesia, compared with E21R (Fig. $1 C)$. These results indicate that GM-CSF is indeed involved in bone cancer pain development, which is in agreement with a previous report (Schweizerhof et al., 2009) that bone cancer pain was attenuated following a specific knockdown of GM-CSF receptors in L4-L5 DRGs of mice.

To further attest that GM-CSF is proalgesic and the primary afferent sensory nerve are the targeted sites of GM-CSF action, we evaluated the effect of direct focal GM-CSF infusion into the L5 DRG on pain-related behavior in naive rats (Fig. 1D). Compared with the vehicle-treated rats, GM-CSF induced significant dosedependent thermal and mechanical hyperalgesia, which persisted for at least $24 \mathrm{~h}$ after injection. Indeed, focal injection of GM-CSF (20-200 ng) via the DRG cannula significantly increased the sensitivity of rats to thermal and mechanical stimuli as measured with the Hargreaves and von Frey tests, respectively $(p<0.05)$. 
Table 2. Summarized effects of GM-CSF on parameters of action potential

\begin{tabular}{|c|c|c|c|c|c|c|c|c|c|}
\hline & $\begin{array}{l}\text { Rhobase currents } \\
\text { (pA) }\end{array}$ & $\mathrm{TP}(\mathrm{mV})$ & $\begin{array}{l}\text { AP amplitude } \\
(\mathrm{mV})\end{array}$ & $\begin{array}{l}\text { Depolarization } \\
\text { rate }(\mathrm{V} / \mathrm{s})\end{array}$ & $\begin{array}{l}\text { AHP amplitude } \\
(\mathrm{mV})\end{array}$ & $\begin{array}{l}\text { AHP duration } \\
\text { (ms) }\end{array}$ & $\mathrm{RMP}(\mathrm{mV})$ & $\begin{array}{l}\text { M-type } \mathrm{K}^{+} \\
\text {current }(\mathrm{pA} / \mathrm{pF})\end{array}$ & $\operatorname{Rin}(M \Omega)$ \\
\hline Control & $198.6 \pm 9.8(63)$ & $-18.6 \pm 0.5(23)$ & $107.9 \pm 2.2(19)$ & $16.2 \pm 0.6(19)$ & $21.3 \pm 0.8(23)$ & $19.4 \pm 0.8(18)$ & $55.1 \pm 0.9(43)$ & $3.93 \pm 1.0(9)$ & $564.0 \pm 51.9(32)$ \\
\hline GM-CSF & $83.1 \pm 2.5^{* *}(39)$ & $-20.4 \pm 0.6^{*}(22)$ & $113.5 \pm 1.8(22)$ & $19.3 \pm 0.4^{*}(21)$ & $21.6 \pm 0.8(26)$ & $21.6 \pm 1.3(18)$ & $55.4 \pm 0.9(58)$ & $3.54 \pm 0.9(8)$ & $504.5 \pm 24.1^{*}(25)$ \\
\hline
\end{tabular}

The nociceptive responses in rats that received $2 \mathrm{ng}$ of GM-CSF also showed a tendency toward sensitization, but these effects did not reach statistical significance.

\section{GM-CSF increased the excitability of small-sized DRG neurons}

The fact that GM-CSF enhanced pain sensitivity when injected into DRGs suggests that GM-CSF might directly sensitize nociceptors by increasing their excitability. To test this hypothesis, we performed current-clamp recordings from the cultured DRG neurons in control conditions and after $24 \mathrm{~h}$ treatment with GMCSF $(200 \mathrm{ng} / \mathrm{ml})$. For this study, small-diameter $(<25 \mu \mathrm{M})$ DRG neurons were selected as these are predominantly nociceptors (Zheng et al., 2013). AP firing was induced by trains of depolarizing current steps from +50 to $+500 \mathrm{pA}$, injected with a $50 \mathrm{pA}$ increment. GM-CSF significantly increased the number of APs induced by the depolarizing current pulses from 100 to $500 \mathrm{pA}$ (Fig. 2A,B). GM-CSF also significantly lowered the rheobase currents (i.e., depolarization current thresholds for eliciting the first action potential) from $198.6 \pm 9.8 \mathrm{pA}(n=63)$ to $83.1 \pm 2.5 \mathrm{pA}$ $(n=39, p<0.05)$; the action potential threshold voltage (i.e., $\mathrm{TP})$ was also significantly decreased from $-18.7 \pm 0.5 \mathrm{mV}(n=$ $23)$ to $-20.4 \pm 0.6 \mathrm{mV}(n=22, p<0.05)$. However, the RMP was not significantly changed (Figs. $1 D, 2 C$ ). We also measured the effect of GM-CSF treatment on other properties of evoked APs such as AP amplitude $(\mathrm{mV})$ and rate of depolarization $(\mathrm{V} / \mathrm{s})$, which are summarized in Table 2. Together, the above data indicate that GM-CSF increases the intrinsic neuronal excitability of primary sensor neurons associated with pain.

\section{GM-CSF increases activity and expression level of Nav1.7, Nav1.8, and Nav1.9 sodium channels}

The sensitizing effect of a single focal in vivo injection of GM-CSF was long lasting (Fig. 1E), and, in addition, intracellular action of GM-CSFRs has long been linked to transcriptional effects via the activation of the Jak-Stat pathway. Thus, we hypothesized that the sensitizing effect of this growth factor might be mediated by changes in the expression of some intrinsic regulators of excitability. Thus, to further explore the mechanisms for GM-CSFinduced hyperactivity of DRG nociceptors, we screened the effect of GM-CSF on ion channels, which have been implicated in the modulation of resting excitability of DRG neurons (Liu et al., 2010; Zheng et al., 2013; Qiu et al., 2016; Isensee et al., 2017; Du et al., 2018). We tested the effect of the treatment of cultured DRG neurons with $200 \mathrm{ng} / \mu \mathrm{l} \mathrm{GM-CSF}(24 \mathrm{~h})$ on the mRNA abundance of the following ion channel genes: $S c n 9 a$ (Nav1.7), Scn10a (Nav1.8), Scn9a (Nav1.9), Kcnd2 (Kv4.2), Ano1 (TMEM16A), P2rx3 (P2X3), Kcng2 (Kv7.2), and Kcnq3 (Kv7.3). Among the transcripts tested, only the mRNAs of voltage-gated sodium channels Nav1.7, Nav1.8, and Nav1.9 were elevated (Fig. 3A). Additionally, the mRNAs of Nav1.7-Nav1.9 expression were elevated by day 7 in DRGs from bone cancer rats relative to those from sham controls (Fig. 3B).
Next, we examined the effects of GM-CSF treatment on the current density of voltage-gated sodium currents in DRG neurons. Total, TTX-S, and TTX- $\mathrm{R} \mathrm{Na}^{+}$currents were recorded (see Materials and Methods) using whole-cell patch clamp. After pretreatment of DRG neurons with GM-CSF (200 ng/ml; $24 \mathrm{~h})$, the peak current density of total $\mathrm{Na}^{+}$currents was increased from $-101.0 \pm 8.8 \mathrm{pA} / \mathrm{pF}(n=44)$ to $-155.8 \pm 9.9 \mathrm{pA} / \mathrm{pF}(n=32)$ at $-10 \mathrm{mV}$; the peak current density of TTX-S currents (mainly Nav1.7 currents) was increased from $-58.0 \pm 6.5 \mathrm{pA} / \mathrm{pF}(n=66)$ to $-100.6 \pm 4.7 \mathrm{pA} / \mathrm{pF}(n=43)$ at $-20 \mathrm{mV}$; the peak current density of TTX-R currents was increased from $-56.5 \pm 4.9$ $\mathrm{pA} / \mathrm{pF}(n=66)$ to $-75.5 \pm 5.7 \mathrm{pA} / \mathrm{pF}(n=43)$ at $-10 \mathrm{mV}$ (Fig. 3C).

The TTX- $\mathrm{R} \mathrm{Na}^{+}$currents were further separated into Nav1.8rich currents and Nav1.9-rich current fractions (see Materials and Methods). After pretreatment of DRG neurons with GMCSF, the peak current density of Nav1.8-rich current fraction was increased from $-44.5 \pm 4.5 \mathrm{pA} / \mathrm{pF}(n=55)$ to $-60.1 \pm 4.4$ $\mathrm{pA} / \mathrm{pF}(n=36)$ at $0 \mathrm{mV}$; the Nav1.9-rich current fraction was increased from $-20.2 \pm 1.4 \mathrm{pA} / \mathrm{pF}(n=53)$ to $-29.4 \pm 0.9$ $\mathrm{pA} / \mathrm{pF}(n=36)$ at $-20 \mathrm{mV}$ (Fig. $3 C)$. In sum, GM-CSF significantly increased the current amplitudes of nociceptor-specific Nav1.7, Nav1.8, and Nav1.9 currents. Furthermore, the protein expression levels of Nav1.7, Nav1.8, and Nav1.9 channels in DRG neurons were also significantly increased after incubation with GM-CSF (200 ng/ml; 18 h; Fig. 3D).

\section{Downregulation of Nav1.7-Nav1.9 channels alleviates GM-CSF-induced pain}

To investigate whether the upregulation of Nav1.7, Nav1.8, and Nav1.9 channels contributes to the GM-CSF-mediated pain, we performed unilateral in vivo knockdown of individual sodium channel subunit in rats using the AS ODNs. AS ODNs against Scn9a, Scn10a, and Scn11a (or a control mismatched ODN) were injected into the L5 DRGs via the DRG cannula to offset the upregulation of these sodium channels, and then the effect of GM-CSF on pain behavior was examined. The knock-down efficiency was measured first; for this, the L5 DRGs were extracted following focal injection of saline, GM-CSF, AS ODN + saline, and AS ODN + GM-CSF, and then the mRNA expression levels of $\operatorname{Scn} 9 a, S c n 10 a$, and $S c n 11 a$ were analyzed by quantitative PCR. In agreement with previous data, the mRNA expression levels of Scn9a, Scn10a, and Scn11a in DRG neurons were significantly increased after GM-CSF injection, and, importantly, these increases were totally reversed by respective AS ODNs (Fig. 4A). Consistent with our earlier conclusion that the upregulation of Na1.7-Nav1.9 channels is a crucial factor in GM-CSF-induced hypersensitivity, AS ODNs against $S c n 9 a, S c n 10 a$, and Scn11a significantly alleviated both mechanical and thermal hypersensitivity developed after the focal DRG application of GM-CSF (Fig. $4 B, C)$. 

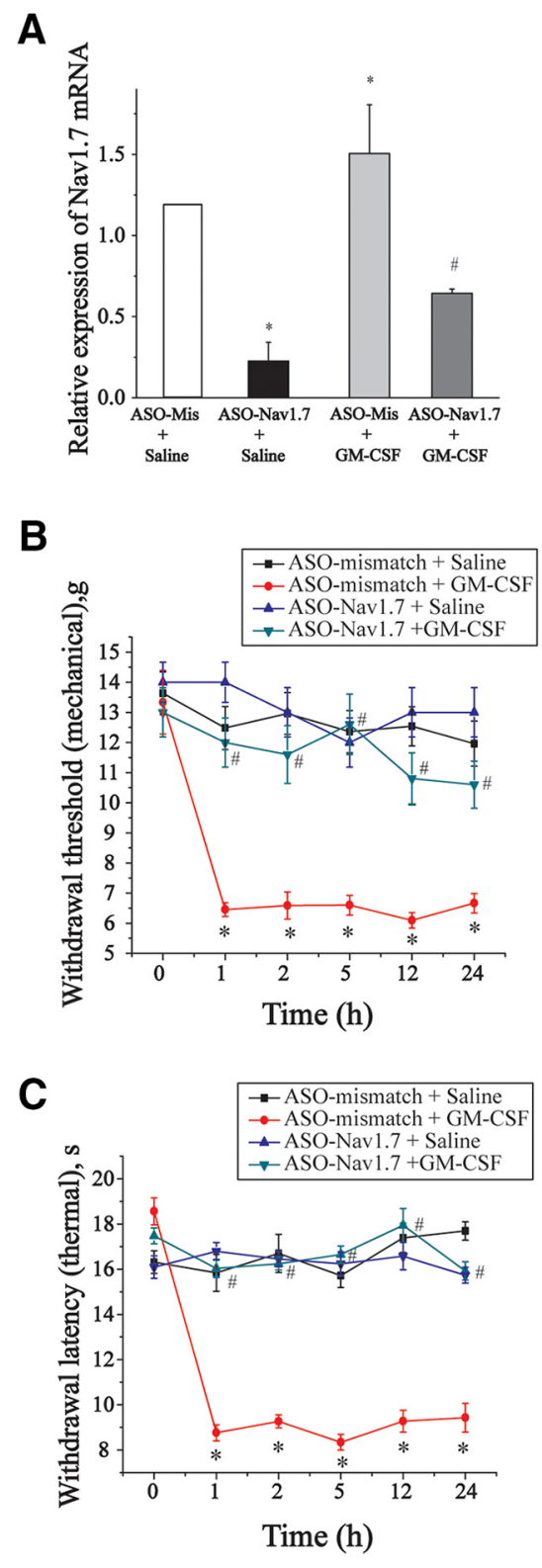
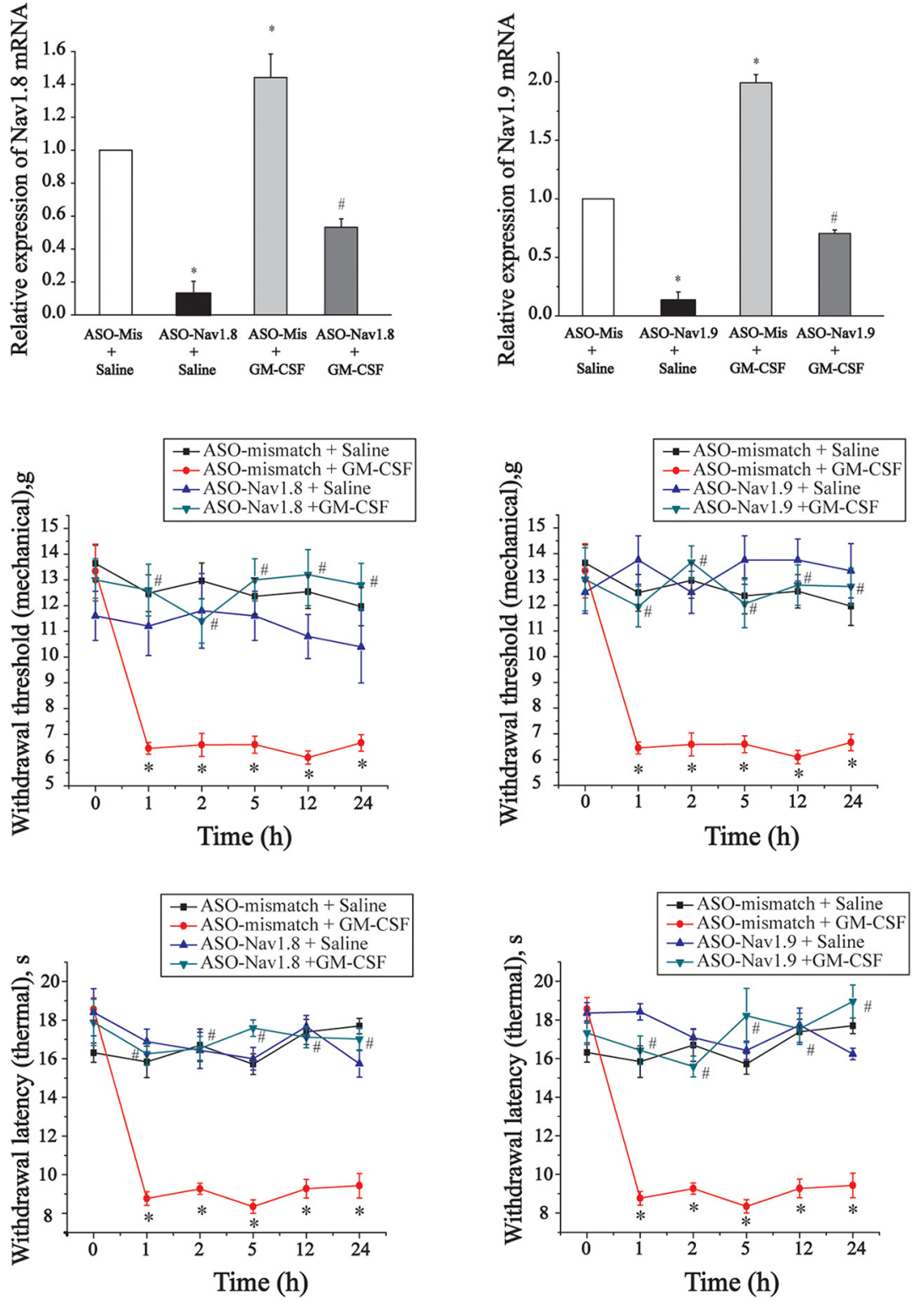

Figure 4. Downregulation of Nav1.7, Nav1.8, and Nav1.9 reverses nociceptive behavior evoked by GM-CSF. $\boldsymbol{A}-\boldsymbol{C}$, Application of AS ODNs (AS0s) in DRGs against Nav1.7, Nav1.8, and Nav1.9 (each ASO, $12.5 \mu \mathrm{g} / \mathrm{rat}, 5 \mu \mathrm{l})$ significantly reduced the mRNA expression level of Nav1.7, Nav1.8, and Nav1.9 increased by GM-CSF treatment (A), and alleviated mechanical (B) and thermal hyperalgesia $(\boldsymbol{C})$ produced by the focal GM-CSF (200 ng) application. For $\boldsymbol{A}: n=6$; unpaired $t$ test, ${ }^{*} p<0.05$ compared with control; \#p $<0.05$ with respect to the corresponding GM-CSF. For $\boldsymbol{B}$, Left, tw0-way ANOVA followed by Bonferroni post hoc tests revealed a significant effect of treatment $\left(F_{(3,305)}=109.59, p=0\right)$, but not time $\left(F_{(4,305)}=0.78, p=0.54\right)$ orinteraction between the two $\left(F_{(12,305)}=0.65\right.$, $p=0.80)$. Middle, There was a significant effect of treatment $\left(F_{(3,305)}=80.53, p=0\right)$, but not time $\left(F_{(4,305)}=0.20, p=0.94\right)$ or interaction between the two $\left(F_{(12,305)}=0.42, p=0.95\right)$. Right, There was a significant effect of treatment $\left(F_{(3,335)}=109.87, p=0\right)$, but not time $\left(F_{(4,335)}=0.89, p=0.47\right)$ or interaction between the two $\left(F_{(12,335)}=0.37, p=0.97\right)$. For $C$, Left, Tw0-way ANOVA followed by Bonferroni post hoc tests revealed a significant effect of treatment $\left(F_{(3,295)}=168.25, p=0\right)$ and interaction between treatment and time $\left(F_{(12,295)}=3.73, p<0.0001\right)$, but the effect of time was not significant $\left(F_{(4,295)}=1.34, p=0.25\right)$. Middle, There was a significant effect of treatment $\left(F_{(3,295)}=336.23, p=0\right)$ and an interaction between treatment and time $\left(F_{(12,295)}=2.04, p=0.02\right)$, but the effect of time was not significant $\left(F_{(4,295)}=1.05, p=0.38\right)$. Right, There was a significant effect of treatment $\left(F_{(3,250)}=274.66, p=0\right)$ and interaction between treatment and time $\left(F_{(12,335)}=5.38, p<0.0001\right)$, but the effect of time was not significant $\left(F_{(4,335)}=0.71, p=0.74\right)$. ${ }^{*} p<0.05$ compared with the vehicle saline; $n=6$, \#p $<0.05$ with respect to the corresponding GM-CSF.

\section{GM-CSF upregulates Nav1.7-Nav1.9 channel expression via the Jak2-Stat3 signaling pathway}

GM-CSF receptor is abundantly expressed in DRGs (Schweizerhof et al., 2009). Thus, we hypothesized that the GM-CSFinduced upregulation of Nav1.7-Nav1.9 in DRG neurons could be mediated by the GM-CSFR and the related cellular signaling pathway. To test this, we focused on the Jak-Stat $3 / 5$ pathway since this is the key pathway for GM-CSF action in hematopoietic cells (Lilly et al., 2001). Activated and phosphorylated states of
Jak1, Jak2, Jak3, Stat3, and Stat5 were first measured in DRG neurons. As shown in Figure 5A, after incubation of DRG cultures with GM-CSF for 25 min, phosphorylated Jak2 and Stat3 were significantly increased, but the level of phosphorylated Jak1 was not changed; phosphorylated Jak3 and Stat5 were not detected. These results indicate that GM-CSF is able to activate the Jak2-Stat3 signaling pathway in DRG neurons. Is this activated Jak2-Stat3 signaling pathway responsible for GM-CSF-induced upregulation of Nav1.7-Nav1.9 in DRG neurons? To test this, the 
A
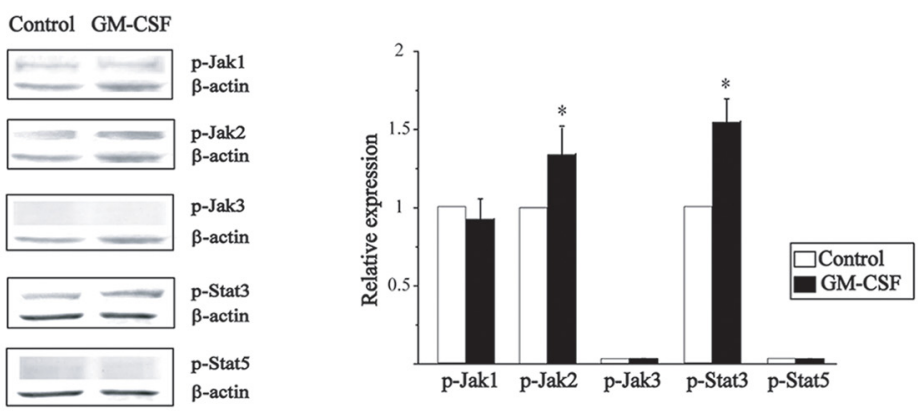

B
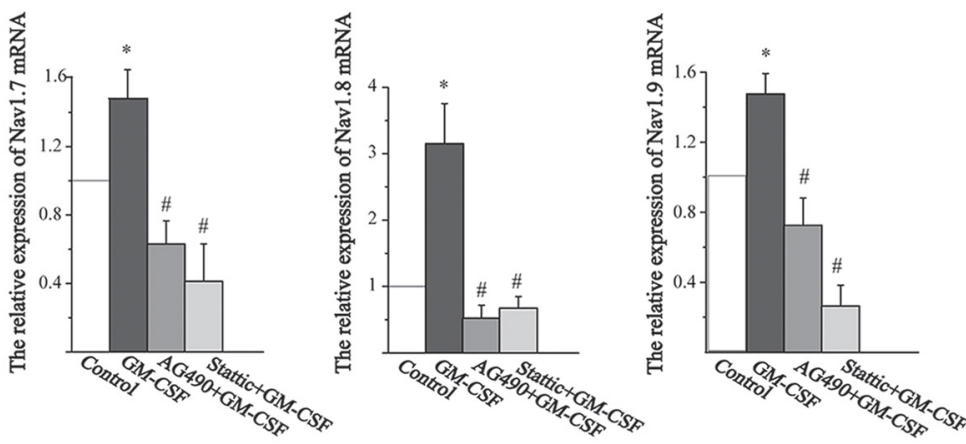

D

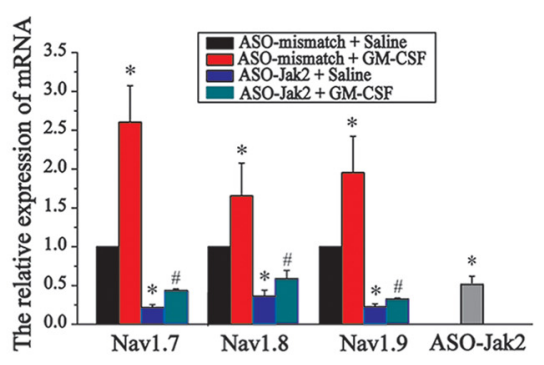

E

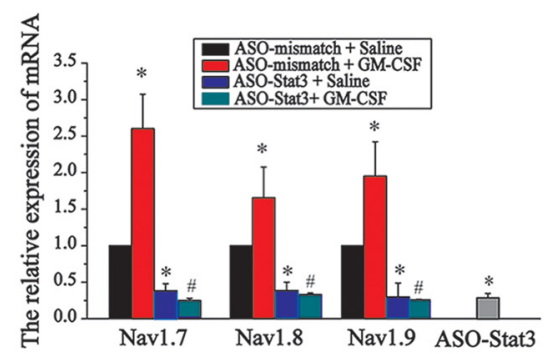

F

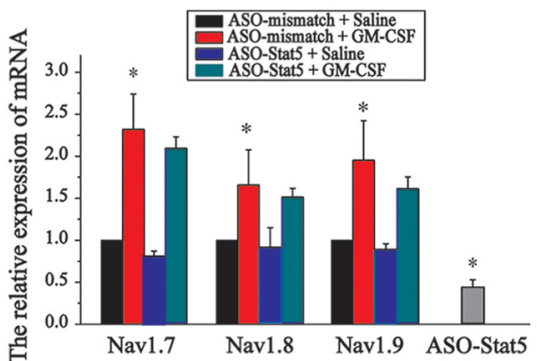

G
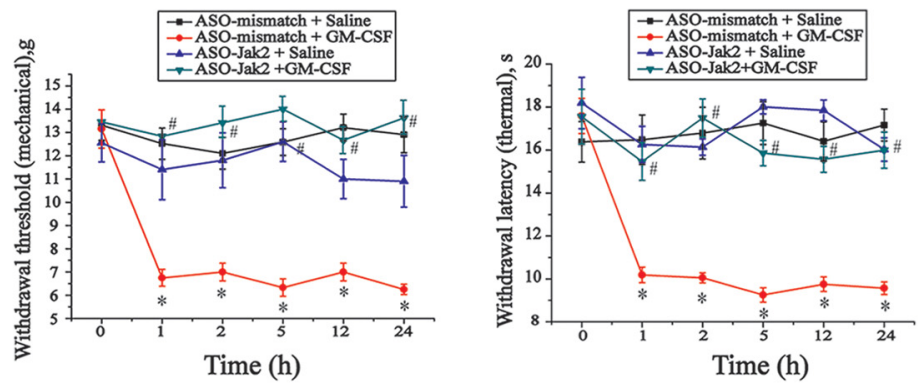

H
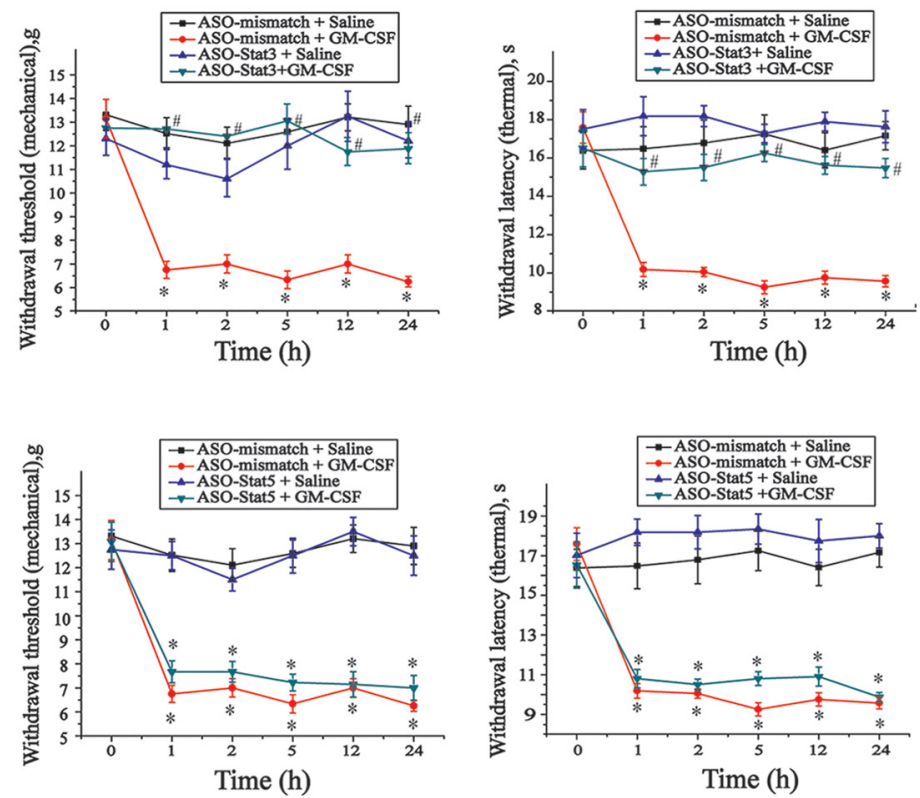

Figure 5. GM-CSF increases the mRNA expression level of Nav1.7, Nav1.8, and Nav1.9 channels through the Jak2-Stat3 signaling pathway. A, Relative expression of p-Jak1, p-Jak2, p-Jak3, p-stat3, and p-stat5 in DRG neurons after incubation with GM-CSF for $25 \min \left(n=3\right.$; unpaired $t$ test, ${ }^{*} p<0.05$ compared with control). $\boldsymbol{B}$, Relative mRNA expression level of Nav1.7, Nav1.8, and Nav1.9 in DRG neurons incubated with GM-CSF in the absence or presence of AG-490 (10 $\mu \mathrm{M})$ and stattic (20 $\mu \mathrm{M})$ for $4 \mathrm{~h}\left(n=4-6\right.$; unpaired $t$ test, ${ }^{*} p<0.05$ compared with control; \#p $<0.05$ with respect to the corresponding GM-CSF). C, Relative Luciferase activity in HEK 293 cells transfected with reporter vector containing Nav1.7, Nav1.8, and (Figure legend continues.) 
acutely disassociated DRG neurons were incubated with GMCSF with and without blockers of Jak2-Stat3 signaling pathway, AG490 $(10 \mu \mathrm{M})$, and Stattic $(20 \mu \mathrm{M})$. Both compounds prevented the upregulation of Nav1.7-Nav1.9 mRNA by GM-CSF (Fig. 5B).

As Stat3 was previously demonstrated to function as a transcriptional activator (Sharma et al., 2018), we thus designed a luciferase reporter assay to determine whether Stat3 acts to regulate Nav1.7-Nav1.9 transcription. Scn9a-Scn11a promoter regions (relative to the transcription start site) were cloned into a luciferase reporter vector (pGL3 Basic plasmid), such that luciferase expression is driven by either of the $S c n 9 a-S c n 11 a$ promoter sequences. We transfected these DNA constructs and either a control pcDNA3.1 plasmid or a pcDNA3.1-stat3 plasmid into HEK293 cells and measured the resulting luciferase activity. Luciferase activity in cells coexpressed with $\operatorname{Scn} 9 \mathrm{a}, \operatorname{Scn} 9 a$, or Scn11 a promoter fragments and Stat 3 was $1.57 \pm 0.14$-fold $(n=$ $4, p<0.05), 1.5 \pm 0.05$-fold $(n=4, p<0.05)$, and $1.5 \pm$ 0.15 -fold $(n=4, p<0.05)$ higher than that in cells coexpressed with Scn9a, Scn9a, or Scn1 la promoter fragments and the control pcDNA3.1 plasmid (Fig. 5C). These results implicate an important role for Stat 3 in promoting Nav1.7, Nav1.8, and Nav1.9 gene expression.

Finally, we assessed whether the downregulation of the Jak2Stat3 signaling pathway would inhibit GM-CSF-induced upregulation of Nav1.7-Nav1.9 and subsequently the pain behaviors. To this end, AS ODNs against Jak2 and Stat3 were injected into DRGs (L5) via a DRG cannula, as described above. AS ODNs against Jak 2 (Fig. 5D) and Stat3 (Fig. 5E), but not against Stat5 (Fig. $5 F$ ), reduced basal mRNA levels of $S c n 9 a-S c n 11 a$ and reversed the GM-CSF-induced upregulation of Scn9a-Scn11a mRNA levels. Consistent with these results, AS ODNs against Jak2 (Fig. 5G) and Stat3 (Fig. 5H), but not AS ODNs against Stat5 (Fig. 5I), prevented the development of the mechanical and thermal hyperalgesia produced by focal in vivo application of GMCSF via the DRG cannula (Fig. 5G-I). AS ODNs against Jak2, Stat3, and Stat5 did not significantly affect the mechanical and thermal sensitivity in rats without GM-CSF treatment (in accord with previous findings suggesting that Nav1.7, Nav1.8, or Nav1.9 knockout or knockdown does not significantly affect threshold

\section{$\leftarrow$}

(Figure legend continued.) Nav1.9 promoter regions (pGL3) coexpressed with either pCDNA3.1 (control) or pcDNA3.1-Stat3 CDNA ( $n=3$; unpaired $t$ test, ${ }^{*} p<0.05$ compared with control). D-F, Relative mRNA level of Nav1.7, Nav1.8, and Nav1.9 in ipsilateral DRGs (L5) of rats receiving AS ODNs (ASOs) against different Jak and Stat signaling molecules $(12.5 \mathrm{mg} / \mathrm{rat}, 5 \mu \mathrm{l}$; $n=6$, unpaired $t$ test, ${ }^{*} p<0.05$ compared with control; $\# p<0.05$ with respect to the corresponding GM-CSF). G-I, Effect of ASOs against Jak and Stat signaling molecules (12.5 $\mathrm{mg} / \mathrm{rat}, 5 \mu \mathrm{l}$ ) on hyperalgesia responses to mechanical and thermal stimuli induced by GM-CSF. ASOs were given through the DRG cannula for $4 \mathrm{~d}$, and then GM-CSF ( $200 \mathrm{ng}$ ) was given. For $\mathbf{G}$, Left, Two-way ANOVA followed by Bonferroni post hoc tests revealed a significant effect of treatment $\left(F_{(3,415)}=125.38, p=0\right)$, but not time $\left(F_{(4,415)}=0.54, p=0.70\right)$ or interaction between the two $\left(F_{(12,415)}=0.73, p=0.73\right)$. Right, There was a significant effect of treatment $\left(F_{(3,425)}=77.18, p=0\right)$, but not time $\left(F_{(4,425)}=1.24, p=0.29\right)$ or interaction between the two $\left(F_{(12,425)}=1.65, p=0.07\right)$. For $\boldsymbol{H}$, Left, Two-way ANOVA followed by Bonferroni post hoc tests revealed a significant effect of treatment $\left(F_{(3,415)}=110.97, p=0\right)$, but not time $\left(F_{(4,415)}=0.38, p=0.82\right)$ or interaction between the two $\left(F_{(12,415)}=0.43, p=0.79\right)$. Right, There was a significant effect of treatment $\left(F_{(3,440)}=115.88, p=0\right)$, but not time $\left(F_{(4,440)}=\right.$ $1.40, p=0.25)$ or interaction between the two $\left(F_{(12,440)}=1.13, p=0.33\right)$. For $I$, Left, Two-way ANOVA followed by Bonferroni post hoc tests revealed a significant effect of treatment $\left(F_{(3,345)}=143.47, p=0\right)$, but not time $\left(F_{(4,415)}=0.74, p=0.57\right)$ or interaction between the two $\left(F_{(12,415)}=0.45, p=0.94\right)$. Right, There was a significant effect of treatment $\left(F_{(3,440)}=\right.$ $111.32, p=0)$, but not time $\left(F_{(4,440)}=0.88, p=0.47\right)$ or an interaction between the two $\left(F_{(12,440)}=0.94, p=0.50\right) . n=6-8 ;{ }^{*} p<0.05$ compared with the vehicle saline; $\# p<0.05$ with respect to the corresponding GM-CSF. sensitivity in mice (Miao et al., 2010; Lolignier et al., 2011; Minett et al., 2014). Together, these results identified Jak2-Stat3mediated upregulation of Nav1.7-Nav1.9 channels as a key signaling pathway involved in the development of GM-CSFinduced pain.

\section{Discussion}

In this study, we demonstrate that GM-CSF promotes bone cancer-associated pain by enhancing the excitability of DRG neurons via the Jak2-Stat3-mediated upregulation of expression of nociceptor-specific voltage-gated sodium channels. First, we show that GM-CSF is highly expressed in osteosarcoma biopsy samples from human patients. Second, we demonstrate that the competitive antagonist of GM-CSF, GM-CSF (E21R), as well as the antibodies against GM-CSF or GM-CSFR are able to reduce both thermal and mechanical hyperalgesia in a rat model of bone cancer. Third, we show that GM-CSF increases the excitability of peripheral nociceptors by upregulating the functional expression of nociceptor-specific $\mathrm{Na}^{+}$channels Nav1.7-Nav1.9. Furthermore, using unilateral in vivo gene knockdown, we further demonstrate that $\mathrm{Na}^{+}$channel upregulation is indeed a necessary step in the development of GM-CSF-induced pain in vivo. Finally, using a set of genetic manipulations and assays, we delineated a molecular mechanism for GM-CSF-induced initiation of pain in bone cancer: upregulation of functional Nav1.7, Nav1.8, and Nav1.9 channel activity through the Jak2-Stat3-mediated activation of Scn $9 a, \operatorname{Scn} 10 a$, and Scn11a gene transcription.

Several recent studies implicated contribution of GM-CSF to different types of pain, including cancer pain, neuropathic, inflammatory, and osteoarthritic pain. (Cook et al., 2012, 2013; Nicol et al., 2018). Yet, the exact mechanism and main molecular steps of the proalgesic action of GM-CSF remained elusive. Our study fills this gap by providing a mechanistic framework for the effect.

Increased excitability of nociceptive neurons is a fundamental mechanism for pain. In turn, changes in excitability are ultimately linked to altered ion channel activity. Thus, in this study we focused on ion channels controlling the excitability of DRG neurons. All results pinpoint Nav.7-Nav1.9 channels as key determinants of the GM-CSF proalgesic action. (1) GM-CSF increased levels of Scn $9 a, \operatorname{Scn} 10 a$, and Scn11a but not the other key ion channels tested. (2) Consistent with the above results, protein levels of Nav1.7-Nav1.9 and the appropriate $\mathrm{Na}^{+}$current fractions in nociceptive DRG neurons were also increased by GM-CSF. (3) Changes in DRG neuron excitability induced by GM-CSF were consistent with elevated $\mathrm{Na}^{+}$channel activity: lowered rheobase, lowered TP, but no significant change in resting membrane potential. Notably, GM-CSF did not change the amplitude of the $\mathrm{M}$-type $\mathrm{K}^{+}$current, which is another type of ion channel that is important for setting the resting excitability parameters of a neuron (Table 2). (4) The downregulation of Nav1.7-Nav1.9 with AS ODNs alleviated GM-CSF-induced pain behavior. While the latter evidence does not directly prove the involvement of these Nav channels in GM-CSF-induced pain specifically (since downregulation of them will probably inhibit any type of pain behavior anyway), the combined evidence implicates the Nav channel mechanism as the most plausible and straightforward explanation for GM-CSF-induced pain nonetheless. However, the contribution of other mechanisms to the GMCSF-induced pain cannot be ruled out at present; indeed, the involvement of other mediators, including Ccl5, Ccl3, and Illa, has been reported (Stosser et al., 2011). 
We provide evidence that Jak2-Stat3 signaling pathway contributes to GM-CSF-mediated upregulation of Nav channels described above and, thus, to hyperalgesia associated with high GM-CSF levels (e.g., as observed in bone cancer). Activation of Jak by GM-CSF leads to the activation of the Stat family transcription factors, which dimerize and translocate to the nucleus and modulate gene expression (Choi et al., 2011). In hematopoietic cells, GM-CSF exerts its biological functions mainly through activation of Jak2, which then activates Stat3 and Stat5, but not Stat2, Stat4, or Stat6 (Zgheib et al., 2013). However, the signal transduction pathways mediated by GM-CSF and its receptors are cell-type specific and may differ significantly (Valdembri et al., 2002). In the present study, we found that in DRG neurons Jak2 and Stat 3 are selectively phosphorylated following the GMCSF treatment; phosphorylated Jak1 was not affected, and phosphorylated Jak3 and Stat 5 were not found at all. Consistent with these results, very low levels of Jak3 and Stat 5 mRNA in DRG neurons were retrieved using the iBrain big data platform ( $\mathrm{Li}$ et al., 2016). Thus, Jak2-Stat3 is likely to be the dominant signaling pathway for GM-CSF to exert its function in DRG neurons.

Luciferase reporter assay provided strong evidence indicating that $S t a t 3$ is able to bind to the promoter regions of $S c n 9 a, S c n 10 a$, and Scn 11a genes to enhance their transcription. In accordance with this observation, the downregulation of Jak2 and Stat 3 with antisense oligodeoxynucleotides reversed the GM-CSF-induced elevation of the mRNA expression level of these Nav channels. Importantly, these antisense oligodeoxynucleotides against Scn9a-Scn11 $a$ also alleviated the GM-CSF-elicited pain behavior. These results not only describe a clear mechanism for how Nav1.7-Nav1.9 channels are upregulated by the GM-CSF signaling pathway, but also indicate that a specific Jak-Stat pathway could be targeted for pain therapeutics.

GM-CSF is used clinically for the treatment of myelodysplastic syndromes, aplastic anemia, tumor radiotherapy, and chemotherapy-induced neutropenia (Garcia et al., 2014).The most severe adverse reaction to these GM-CSF therapies is bone pain, and the incidence is reported to reach up to $90 \%$ (Stosser et al., 2011). These clinical observations align very well with our results showing that GM-CSF induces pain behavior in rats when injected into DRGs at a concentration of $20 \mathrm{ng} / \mathrm{ml}$ GM-CSF, which is lower than the blood concentration of after a single-dose administration of GM-CSF in humans $(\sim 600 \mathrm{ng} / \mathrm{ml}$; Alexander et al., 2016). Thus, clinically administered GM-CSF reaches sufficient blood concentrations to be able to sensitize bone periosteal nerves and nociceptive neurons through the mechanism described here.

In summary, this study provides a mechanistic explanation for the role of GM-CSF in pain, specifically in pain associated with bone cancer and with the GM-CSF-based therapies. This novel mechanism should be considered as a potential target for future pain treatments.

\section{References}

Alexander WS (2016) In vivo at last: demonstrating the biological credentials and clinical potential of GM-CSF. Exp Hematol 44:669-673.

Bali KK, Venkataramani V, Satagopam VP, Gupta P, Schneider R, Kuner R (2013) Transcriptional mechanisms underlying sensitization of peripheral sensory neurons by granulocyte-/granulocyte-macrophage colony stimulating factors. Mol Pain 9:48.

Blanchard MG, Rash LD, Kellenberger S (2012) Inhibition of voltage-gated $\mathrm{Na}(+)$ currents in sensory neurones by the sea anemone toxin APETx2. Br J Pharmacol 165:2167-2177.

Chaplan SR, Bach FW, Pogrel JW, Chung JM, Yaksh TL (1994) Quantitative assessment of tactile allodynia in the rat paw. J Neurosci Methods 53:5563.
Cheng X, Dib-Hajj SD, Tyrrell L, Te Morsche RH, Drenth JP, Waxman SG (2011) Deletion mutation of sodium channel $\mathrm{Na}(\mathrm{V}) 1.7$ in inherited erythromelalgia: enhanced slow inactivation modulates dorsal root ganglion neuron hyperexcitability. Brain 134:1972-1986.

Choi JK, Kim KH, Park H, Park SR, Choi BH (2011) Granulocyte macrophage-colony stimulating factor shows anti-apoptotic activity in neural progenitor cells via JAK/STAT5-bcl-2 pathway. Apoptosis 16:127-134.

Choi JS, Dib-Hajj SD, Waxman SG (2007) Differential slow inactivation and use-dependent inhibition of Nav1.8 channels contribute to distinct firing properties in IB4+ and IB4- DRG neurons. J Neurophysiol 97:1258-1265.

Cook AD, Pobjoy J, Steidl S, Dürr M, Braine EL, Turner AL, Lacey DC, Hamilton JA (2012) Granulocyte-macrophage colony-stimulating factor is a key mediator in experimental osteoarthritis pain and disease development. Arthritis Res Ther 14:R199.

Cook AD, Pobjoy J, Sarros S, Steidl S, Dürr M, Lacey DC, Hamilton JA (2013) Granulocyte-macrophage colony-stimulating factor is a key mediator in inflammatory and arthritic pain. Ann Rheum Dis 72:265-270.

Cummins TR, Black JA, Dib-Hajj SD, Waxman SG (2000) Glial-derived neurotrophic factor upregulates expression of functional SNS and NaN sodium channels and their currents in axotomized dorsal root ganglion neurons. J Neurosci 20:8754-8761.

de Mooij T, Wuyts W, Ham J (2012) Phenotypic differences in multiple osteochondromas in monozygotic twins: a case report. JBJS Case Connect 2:e60.

Dib-Hajj SD, Estacion M, Jarecki BW, Tyrrell L, Fischer TZ, Lawden M, Cummins TR, Waxman SG (2008) Paroxysmal extreme pain disorder M1627K mutation in human Nav1.7 renders DRG neurons hyperexcitable. Mol Pain 4:37.

Du X, Hao H, Gigout S, Huang D, Yang Y, Li L, Wang C, Sundt D, Jaffe DB, Zhang H, Gamper N (2014) Control of somatic membrane potential in nociceptive neurons and its implications for peripheral nociceptive transmission. Pain 155:2306-2322.

Du X, Hao H, Yang Y, Huang S, Wang C, Gigout S, Raml R, Li X, Jaworska E, Edwards I, Deuchars J, Yanagawa Y, Qi J, Guan B, Jaffe DB, Zhang H, Gamper N (2017) Local GABAergic signaling within sensory ganglia controls peripheral nociceptive transmission. J Clin Invest 127:17411756.

Du X, Gao H, Jaffe D, Zhang H, Gamper N (2018) M-type K(+) channels in peripheral nociceptive pathways. Br J Pharmacol 175:2158-2172.

Fortin CF, Larbi A, Dupuis G, Lesur O, Fülöp T Jr (2007) GM-CSF activates the Jak/STAT pathway to rescue polymorphonuclear neutrophils from spontaneous apoptosis in young but not elderly individuals. Biogerontology 8:173-187.

François-Moutal L, Dustrude ET, Wang Y, Brustovetsky T, Dorame A, Ju W, Moutal A, Perez-Miller S, Brustovetsky N, Gokhale V, Khanna M, Khanna R (2018) Inhibition of the Ubc9 E2 SUMO conjugating enzyme-CRMP2 interaction decreases Nav1.7 currents and reverses experimental neuropathic pain. Pain 159:2115-2127.

Garcia JA, Elson P, Tyler A, Triozzi P, Dreicer R (2014) Sargramostim (GM$\mathrm{CSF}$ ) and lenalidomide in castration-resistant prostate cancer (CRPC): results from a phase I-II clinical trial. Urol Oncol 32:33.e11-7.

Huang J, Han C, Estacion M, Vasylyev D, Hoeijmakers JG, Gerrits MM, Tyrrell L, Lauria G, Faber CG, Dib-Hajj SD, Merkies IS, Waxman SG (2014) Gain-of-function mutations in sodium channel $\mathrm{Na}(\mathrm{v}) 1.9$ in painful neuropathy. Brain 137:1627-1642.

Huang J, Vanoye CG, Cutts A, Goldberg YP, Dib-Hajj SD, Cohen CJ, Waxman SG, George AL Jr (2017) Sodium channel NaV1.9 mutations associated with insensitivity to pain dampen neuronal excitability. J Clin Invest 127:2805-2814.

Isensee J, Krahé L, Moeller K, Pereira V, Sexton JE, Sun X, Emery E, Wood JN, Hucho T (2017) Synergistic regulation of serotonin and opioid signaling contributes to pain insensitivity in Nav1.7 knockout mice. Sci Signal 10:eaah4874.

Iversen PO, Rodwell RL, Pitcher L, Taylor KM, Lopez AF (1996) Inhibition of proliferation and induction of apoptosis in juvenile myelomonocytic leukemic cells by the granulocyte-macrophage colony-stimulating factor analogue E21R. Blood 88:2634-2639.

Jarecki BW, Piekarz AD, Jackson JO 2nd, Cummins TR (2010) Human voltage-gated sodium channel mutations that cause inherited neuronal 
and muscle channelopathies increase resurgent sodium currents. J Clin Invest 120:369-378.

Lai J, Gold MS, Kim CS, Bian D, Ossipov MH, Hunter JC, Porreca F (2002) Inhibition of neuropathic pain by decreased expression of the tetrodotoxin-resistant sodium channel, NaV1.8. Pain 95:143-152.

Li CL, Li KC, Wu D, Chen Y, Luo H, Zhao JR, Wang SS, Sun MM, Lu YJ, Zhong YQ, Hu XY, Hou R, Zhou BB, Bao L, Xiao HS, Zhang X (2016) Somatosensory neuron types identified by high-coverage single-cell RNA-sequencing and functional heterogeneity. Cell Res 26:83-102.

Li Y, North RY, Rhines LD, Tatsui CE, Rao G, Edwards DD, Cassidy RM, Harrison DS, Johansson CA, Zhang H, Dougherty PM (2018) DRG voltagegated sodium channel 1.7 is upregulated in paclitaxel-induced neuropathy in rats and in humans with neuropathic pain. J Neurosci 38:1124-1136.

Lilly MB, Zemskova M, Frankel AE, Salo J, Kraft AS (2001) Distinct domains of the human granulocyte-macrophage colony-stimulating factor receptor alpha subunit mediate activation of Jak/Stat signaling and differentiation. Blood 97:1662-1670.

Liu B, Linley JE, Du X, Zhang X, Ooi L, Zhang H, Gamper N (2010) The acute nociceptive signals induced by bradykinin in rat sensory neurons are mediated by inhibition of M-type $\mathrm{K}+$ channels and activation of Ca2+-activated cl- channels. J Clin Invest 120:1240-1252.

Lolignier S, Amsalem M, Maingret F, Padilla F, Gabriac M, Chapuy E, Eschalier A, Delmas P, Busserolles J (2011) Nav1.9 channel contributes to mechanical and heat pain hypersensitivity induced by subacute and chronic inflammation. PLoS One 6:e23083.

Miao XR, Gao XF, Wu JX, Lu ZJ, Huang ZX, Li XQ, He C, Yu WF (2010) Research article bilateral downregulation of Nav1.8 in dorsal root ganglia of rats with bone cancer pain induced by inoculation with walker 256 breast tumor cells. BMC Cancer 10:216.

Minett MS, Falk S, Santana-Varela S, Bogdanov YD, Nassar MA, Heegaard AM, Wood JN (2014) Pain without nociceptors? Nav1.7-independent pain mechanisms. Cell Rep 6:301-312.

Nicol LSC, Thornton P, Hatcher JP, Glover CP, Webster CI, Burrell M, Hammett K, Jones CA, Sleeman MA, Billinton A, Chessell I (2018) Central inhibition of granulocyte-macrophage colony-stimulating factor is analgesic in experimental neuropathic pain. Pain 159:550-559.
Qiu F, Li Y, Fu Q, Fan YY, Zhu C, Liu YH, Mi WD (2016) Stromal cellderived factor 1 increases tetrodotoxin-resistant sodium currents Nav1.8 and Nav1.9 in rat dorsal root ganglion neurons via different mechanisms. Neurochem Res 41:1587-1603.

Schweizerhof M, Stösser S, Kurejova M, Njoo C, Gangadharan V, Agarwal N, Schmelz M, Bali KK, Michalski CW, Brugger S, Dickenson A, Simone DA, Kuner R (2009) Hematopoietic colony-stimulating factors mediate tumor-nerve interactions and bone cancer pain. Nat Med 15:802-807.

Sharma A, Oishi N, Boddicker RL, Hu G, Benson HK, Ketterling RP, Greipp PT, Knutson DL, Kloft-Nelson SM, He R, Eckloff BW, Jen J, Nair AA, Davila JI, Dasari S, Lazaridis KN, Bennani NN, Wu TT, Nowakowski GS, Murray JA, et al. (2018) Recurrent STAT3-JAK2 fusions in indolent T-cell lymphoproliferative disorder of the gastrointestinal tract. Blood 131:2262-2266.

Stosser S, Schweizerhof M, Kuner R (2011) Hematopoietic colonystimulating factors: new players in tumor-nerve interactions. J Mol Med (Berl) 89:321-329.

Valdembri D, Serini G, Vacca A, Ribatti D, Bussolino F (2002) In vivo activation of JAK2/STAT-3 pathway during angiogenesis induced by GMCSF. FASEB J 16:225-227.

Wang LN, Yao M, Yang JP, Peng J, Peng Y, Li CF, Zhang YB, Ji FH, Cheng H, Xu QN, Wang XY, Zuo JL (2011) Cancer-induced bone pain sequentially activates the ERK/MAPK pathway in different cell types in the rat spinal cord. Mol Pain 7:48.

Yoneda T, Hiasa M, Nagata Y, Okui T, White F (2015) Contribution of acidic extracellular microenvironment of cancer-colonized bone to bone pain. Biochim Biophys Acta 1848:2677-2684.

Zgheib A, Pelletier-Bonnier É, Levros LC Jr, Annabi B (2013) Selective JAK/ STAT3 signalling regulates transcription of colony stimulating factor-2 and -3 in concanavalin-A-activated mesenchymal stromal cells. Cytokine 63:187-193.

Zheng Q, Fang D, Liu M, Cai J, Wan Y, Han JS, Xing GG (2013) Suppression of $\mathrm{KCNQ} / \mathrm{M}(\mathrm{Kv} 7)$ potassium channels in dorsal root ganglion neurons contributes to the development of bone cancer pain in a rat model. Pain 154:434-448. 